Research Paper

\title{
Early cortical metabolic rearrangement related to clinical data in idiopathic sudden sensorineural hearing loss
}

\author{
Alessandro Micarelli a, b, *, 1 , Agostino Chiaravalloti ${ }^{c, 1}$, Andrea Viziano ${ }^{\text {a }}$, Roberta Danieli ${ }^{\text {, }}$ \\ Orazio Schillaci ${ }^{c, d}$, Marco Alessandrini ${ }^{\text {a }}$ \\ ${ }^{a}$ University of Rome Tor Vergata, Department of Clinical Sciences and Translational Medicine, Otolaryngology Unit, Italy \\ ${ }^{\mathrm{b}}$ University of Rome Tor Vergata, Department of Systems Medicine, Neuroscience Unit, Italy \\ ${ }^{\mathrm{c}}$ University of Rome Tor Vergata, Department of Biomedicine and Prevention, Nuclear Medicine Unit, Italy \\ d IRCCS Neuromed, Pozzilli, Italy
}

\section{A R T I C L E I N F O}

\section{Article history:}

Received 19 December 2016

Received in revised form

13 April 2017

Accepted 23 April 2017

Available online 25 April 2017

\section{Keywords:}

Sudden hearing loss

Positron emission tomography

Auditory scene analysis network

Frontoparietal control system

Tinnitus

Speech discrimination score

\begin{abstract}
A B S T R A C T
Results in studies concerning cortical changes in idiopathic sudden sensorineural hearing loss (ISSNHL) are not homogeneous, in particular due to the different neuroimaging techniques implemented and the diverse stages of ISSNHL studied. Considering the recent advances in state-of-the-art positron emission tomography (PET) cameras, the aim of this study was to gain more insight into the neuroanatomical differences associated with the earliest stages of unilateral ISSNHL and clinical-perceptual performance changes.

After an audiological examination including the mean auditory threshold (mean AT), mean speech discrimination score (mean SDS) and Tinnitus Handicap Inventory (THI), 14 right-handed ISSNHL patients underwent brain $\left[{ }^{18} \mathrm{~F}\right]$ fluorodeoxyglucose (FDG)-PET within $72 \mathrm{~h}$ of the onset of symptoms. When compared to an homogeneous group of 35 healthy subjects by means of statistical parametric mapping, a relative increase in FDG uptake was found in the right superior and medial frontal gyrus as well as in the right anterior cingulate cortex in ISSNHL patients. Conversely, the same group showed a significant relative decrease in FDG uptake in the right middle temporal, precentral and postcentral gyrus as well as in the left posterior cingulate cortex, left lingual, superior, middle temporal and middle frontal gyrus and in the left insula. Regression analysis showed a positive correlation between mean THI and glucose consumption in the right anterior cingulate cortex and a positive correlation between mean SDS and glucose consumption in the left precentral gyrus.

The relative changes in FDG uptake found in these brain regions and the positive correlation with mean SDS and THI scores in ISSNHL could possibly highlight new aspects of cerebral rearrangement, contributing to further explain changes in those functions that support speech recognition during the sudden impairment of unilateral auditory input.
\end{abstract}

(c) 2017 Elsevier B.V. All rights reserved.

\section{Introduction}

Sudden sensorineural hearing loss (SSNHL) is defined as a unilateral loss of hearing occurring over $24-72 \mathrm{~h}$ with a loss of $30 \mathrm{~dB}$ or more in at least 3 contiguous frequencies on pure-tone audiogram (Alexander and Harris, 2013). In line with the uncertainties of its

\footnotetext{
* Corresponding author. University of Rome Tor Vergata, Department of Clinical Sciences and Translational Medicine, Via Montpellier, 1, $6^{\circ}$ floor, E Sud Tower, 00133, Rome, Italy.

E-mail address: alessandromicarelli@yahoo.it (A. Micarelli).

1 These authors contributed equally to this study.
}

etiopathogenesis (Capuano et al., 2015), detailed investigations based on medical and physical examinations, audiovestibular tests, blood examinations and brain magnetic resonance imaging (MRI) studies have not been able to identify a specific cause in over $70 \%$ of patients, which are therefore (Alexander and Harris, 2013; Berrettini et al., 2013) considered cases of idiopathic SSNHL (ISSNHL).

Although the involvement of the hearing function in SSNHL patients is usually unilateral with no side of preference (Fan et al., 2015), these patients' hearing often does not even improve after high-quality appropriate therapy (Fan et al., 2015). This may indicate that some kind of irreversible structural brain damage has 
already occurred in these SSNHL patients (Fan et al., 2015).

Moreover, diverse brain structural changes have been reported in patients with different kinds of auditory impairment such as tinnitus, unilateral hearing loss, or deafness (Boyen et al., 2013; Fan et al., 2015; Profant et al., 2014; Vanneste et al., 2015; Yang et al., 2014), and results among ISSNHL are not homogeneous. This aspect could be related to many experimental factors such as the sample age and the side of the hearing impairment and especially to disease duration and neuroimaging techniques (Bilecen et al., 2000; Fan et al., 2015; Yang et al., 2014). Regarding this, up to now, neither neuroimaging studies have been implemented in the very first hours of the disease nor have metabolic techniques been used in order to discover possible ISSNHL-related cortical changes. In fact - amongst others - studies of the auditory system during ISSNHL have been conducted exclusively by means of MRI-related techniques (Berding et al., 2015; Boyen et al., 2013; Fan et al., 2015; Yang et al., 2014).

Starting from the 1980s, $\left[{ }^{18} \mathrm{~F}\right]$ fluorodeoxyglucose (FDG) positron emission tomography (PET)/computer tomography (CT) has been used as a valuable tool for functional neuroanatomy imaging (Berding et al., 2015; Frackowiak and Friston, 1994) and many studies consider that it offers considerable advantages when studying the auditory system (Berding et al., 2015; Talavage et al., 2014). In fact, it has been demonstrated as a quiet and reliable imaging technique for overcoming possible unintended sensory stimulations (Alessandrini et al., 2014a, 2016; Benjamin et al., 2010; Gaab et al., 2008). Other neuroimaging techniques (i.e., MRI) - due to time-consuming and noisy settings (Cardier et al., 2015) - may have constituted a possible bias to the interpretation of the majority of existing data (Alessandrini et al., 2016). Therefore, it remains unclear to what extent discrepancies in the results of different studies reflect unwanted attentional behaviour or the sensoryspecific engagement of different brain regions, rather than methodological differences (Alessandrini et al., 2014a, 2016).

Although some studies have already reported brain atrophy and impaired microstructure induced by unilateral SSNHL (Chang et al., 2004; Lin et al., 2008; Yang et al., 2014), whether these changes had already occurred during the acute period is still unknown (Fan et al., 2015). Thus, considering i) the degree of inconsistency in the literature regarding brain structure alterations during the acute stage of unilateral ISSNHL and ii) the improved sensitivity of the state-of-the-art PET cameras making it possible to create a defined baseline condition for the exploration of auditory default and avoid possible cortical activation related to unwanted attentional processes (Alessandrini et al., 2014a), the aim of the present study was to gain more insight into the neuroanatomical differences that are related to the earliest stages of unilateral ISSNHL (Fan et al., 2015) and particularly to focus on those 'baseline' metabolic and related clinical-perceptual performance changes occurring during the early onset of the disorder.

\section{Materials and methods}

\subsection{Subjects and diagnosis}

Sixteen patients with ISSNHL affecting the right ear were recruited in line with the generally accepted criteria (Alexander and Harris, 2013). We also enrolled a population of gender- and agematched right-handed healthy subjects serving as a control group (HC). Both eligible ISSNHL patients and HC subjects were required to report negative anamnesis for malignancy, head trauma, neuropsychiatric disorders, metabolic, cardiovascular, endocrine, infectious and otoneurological diseases. The peripheral blood of ISSNHL patients and HC subjects was tested for the usual parameters and neurological disturbances were excluded by means of the
Mini Mental State Examination and T2-weighted and/or diffusionweighted brain MRI sequences with a $1.5 \mathrm{~T}$ clinical MRI scanner. Moreover, we excluded all patients in treatment with drugs that could interfere with ${ }^{18} \mathrm{~F}$-FDG uptake and distribution in the brain (Alessandrini et al., 2014b, 2016; Varrone et al., 2009) and with medication possibly affecting inner ear function. Both brain MRI and PET/CT, as well as audiological tests, were performed within $72 \mathrm{~h}$ from the onset of symptoms in all patients before treatment with any drugs. Finally, no patient was pregnant or breastfeeding.

The Ethics Committee of the Tor Vergata University School of Medicine approved the protocol research. The study adhered to the principles of the Declaration of Helsinki and all of the participants provided written informed consent after receiving a detailed explanation of the study.

After collecting a thorough medical history and performing a neuro-otological examination the subjects in both groups underwent the following procedures:

\subsection{Pure tone audiometry}

ISSNHL and HC subjects underwent pure tone audiometry tests (GSI 61 Clinical Audiometer, Grason-Stadler, United States). Subjective auditory thresholds for 125, 250, 500,1000, 2000, 4000 and $8000 \mathrm{~Hz}$ for both ears were included in the study (Micarelli et al., 2016).

\subsection{Tinnitus assessment}

Because most of the ISSNHL patients complained of tinnitus and its prevalence is also increased in the healthy population without a positive history for audiological risk factors, we assessed the tinnitus handicap by means of the Italian version of the Tinnitus Handicap Inventory (THI) questionnaire (Monzani et al., 2008) in both ISSNHL and HC subjects.

\subsection{Speech discrimination score}

We analysed speech perception performance at a comfortable loudness level in both ISSNHL and HC subjects by means of the speech discrimination score (SDS). Tests were performed for both ears alternatively, with the non-tested ear masked with speech noise. We performed a variation of the speech perception protocol used by Berding et al. (2015). All subjects were asked to rate the subjective loudness of a continuous $1000 \mathrm{~Hz}$ pure tone, initially presented at an intensity of $25 \mathrm{~dB}$ above the mean threshold at speech frequencies (Penrod, 1994), on a scale from 1 to 5 , with 1 being a 'very soft' sound and 5 being 'very loud'. We performed speech audiometry at an intensity rated by the subjects as 3 on this scale. A $110 \mathrm{~dB}$ SPL intensity ceiling was established, to avoid acoustic trauma. Speech audiometry consisted in a list of 10 disyllabic, phonetically- and frequency-balanced words, which the subjects had to repeat correctly. Three lists were presented for each subject: the best out of the three performances, multiplied by 10 , represented the subjects' SDS. Lip-reading was ruled out by administering the stimuli with a prerecorded CD connected to the audiometer.

\subsection{PET/CT examination}

The Discovery VCT PET/CT system (GE Medical Systems, Tennessee, USA) was used to assess FDG brain distribution in all subjects by means of a 3D-mode standard technique in a $256 \times 256$ matrix. Reconstruction was performed using the 3-dimensional reconstruction method of ordered-subset expectation maximization (OSEM) with 20 subsets and four iterations. The system 
combines a high-speed ultra 16-detector-row (912 detectors per row), a CT unit and a PET scanner with 10080 bismuth germanate crystals in 24 rings (axial full width at half-maximum $1-\mathrm{cm}$ radius, $5.2 \mathrm{~mm}$ in 3D mode, $157 \mathrm{~mm}$ axial field of view). A low-amperage CT scan of the head for attenuation correction (40 mA; $120 \mathrm{Kv}$ ) was performed before PET image acquisition. All subjects fasted for at least $5 \mathrm{~h}$ before the i.v. FDG infusion; the serum glucose level was a minimum of $95 \mathrm{mg} / \mathrm{ml}$ in all of them. External acoustic stimulation was eliminated by plugging both ears hermetically. All subjects were administered i.v. infusions of $185-210 \mathrm{MBq}$ of FDG and were hydrated with $500 \mathrm{ml}$ of $\mathrm{NaCl}$ 0.9\%. (Alessandrini et al., 2013; Chiaravalloti et al., 2013; Schecklmann et al., 2013; Alessandrini et al., 2014a; Alessandrini et al., 2014b, 2016; Chiaravalloti et al., 2015).

\subsection{Data handling and statistical analysis}

\subsubsection{Audiological tests}

Mean and standard deviations (SD) were calculated for THI and for both auditory threshold (mean AT) and SDS (mean SDS) in both ears in ISSNHL and in HC subjects.

In order to assess that the data were of Gaussian distribution, the D'Agostino K squared normality test was applied (where the null hypothesis is that the data are normally distributed).

A 'between-groups' analysis of variance (ANOVA) was performed for THI, mean PTA and mean SDS measures. Age and gender were handled as continuous and categorical predictors, respectively. The significant cut-off level $(\alpha)$ was set at a p value of 0.01 . A correlation analysis between mean AT, mean SDS and THI was achieved by means of Spearman rank correlation. The Bonferroni correction for multiple comparisons was used as a post-hoc test of both significant main effects of ANOVA and Spearman rank correlation analysis (STATISTICA 7 package for Windows).

\subsubsection{PET/CT data}

We used statistical parametric mapping (SPM8, Wellcome Department of Cognitive Neurology, London, UK) implemented in Matlab R2012b (Mathworks, Natick, MA, USA) for the analysis of $\left[{ }^{18}\right.$ F]FDG PET data. Non-linear spatial normalization allows us to transform the $\left[{ }^{18} \mathrm{~F}\right.$ FDG PET data into the Montreal Neurological Institute space. A specific template for spatial normalization was used (Della Rosa et al., 2014). The spatially normalized set of images was then smoothed with an $8 \mathrm{~mm}$ isotropic Gaussian filter to blur for individual variations in gyral anatomy and to increase the signal-to-noise ratio. Images were globally normalized to 50 using proportional scaling to remove confounding effects of global cerebral glucose consumption changes, with a masking threshold of 0.8 . The resulting statistical parametric maps, SPM[t], were transformed into normal distribution (SPM[z]) unit. Correction of SPM coordinates to match the Talairach coordinates was achieved by the subroutine implemented by Matthew Brett (http://www.mrc-cbu. cam.ac.uk/Imaging). Cerebral areas were identified at a range of 0-3 $\mathrm{mm}$ from the corrected Talairach coordinates of the SPM output isocentres, after importing the corrected coordinates, from the Talairach client (http://www.talairach.org/index.html). In line with Bennett and colleagues (Bennett et al., 2009), SPMt-maps were set at $\mathrm{p}<0.05$, corrected for multiple comparisons with the False Discovery Rate option at voxel level, and at $\mathrm{p}<0.01$ corrected for multiple comparison at cluster level. The clusters containing more than $100(5 \times 5 \times 5$ voxels, i.e. $11 \times 11 \times 11 \mathrm{~mm})$ contiguous voxels were accepted as significant (Alessandrini et al., 2013; Chiaravalloti et al., 2013; Alessandrini et al., 2014a, 2014b; Chiaravalloti et al., 2015; Alessandrini et al., 2016). The ISSNHL versus HC comparison and vice versa were performed by means of the 'two-sample $t$-test' study design model, using age and sex as covariates.

The relationships between FDG distribution in the brain and the results of THI and SDS were evaluated in ISSNHL and HC using a 'regression analysis' design model. The cluster obtained by this analysis was exported and further analysed after a normalization process. In particular, the mean signal intensities computed by each cluster were normalized within each subject to the average intensities of the whole cerebellum volume of interest, as defined by another similar report by our group in this field (Liguori et al., 2016). Normalization based upon activity in the cerebellum, instead of whole brain counts as in the reference region, was more accurate in distinguishing patients from controls in neurodegenerative diseases (Soonawala et al., 2002). As proposed previously by Pagani et al. (2015), a dataset including normalized FDG PET values relevant to the examined cluster was exported. We generated Regions of Interest masks based on the atlas that include the anatomic label (Lancaster et al., 1997, 2000). For this purpose, WFU Pickatlas was used (Lancaster et al., 1997, 2000; Maldjian et al., 2003).

\section{Results}

\subsection{Patients}

Among 16 consecutive right ear ISSNHL patients, 1 reported diabetes and 1 a history of drug addiction and were excluded. Therefore, 14 right-handed ISSNHL patients ( 8 males; 6 females; $48.3 \pm 7.6$ years) met the eligibility criteria and were included in the study. The HC consisted of 35 right-handed gender- and agematched healthy individuals (19 males; 16 females; $49.6 \pm 6.7$ years).

\subsection{Audiological testing}

The mixed Group $\times$ Test ANOVA highlighted a significant Group $\times$ Test interaction $[\mathrm{F}(2,94)=1659, \mathrm{p}<0.01]$ which accounted for the lower level significant main effects of Group $[F(1,47)=258,02$, $(\mathrm{p}=0.0000)]$ and Test $[\mathrm{F}(2,94)=300,62,(\mathrm{p}=0.0000)]$. The significant double interaction was further investigated with post-hoc comparisons, which highlighted a significant $(\mathrm{p}<0.01$, Bonferroni corrected) decrease in right ear mean AT and mean SDS and a significant $(\mathrm{p}<0.01$, Bonferroni corrected) THI score increase was found in ISSNHL patients when compared to HC subjects (Fig. 1). No

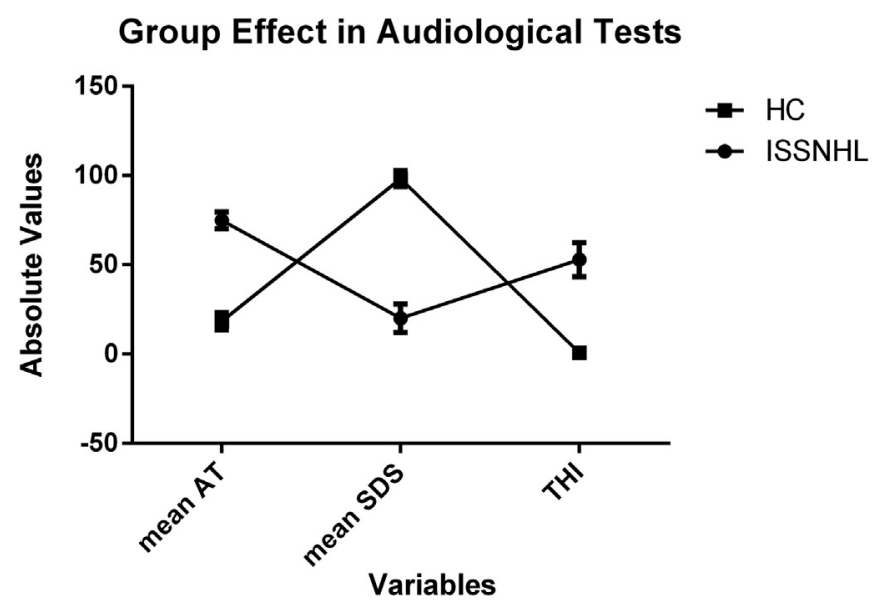

Fig. 1. Significant between-group effect found in Tinnitus Handicap Inventory (THI), mean Speech Discrimination Score (mean SDS) and right mean ear auditory threshold (mean AT) when comparing healthy (HC) and idiopathic sudden sensorineural hearing loss (ISSNHL) subjects. 
statistically significant difference was found between left and right ear mean AT and SDS results in HC.

A significant positive correlation was found in ISSNHL patients between mean right AT and THI $(r=0.95, p<0.01$, Bonferroni corrected) and negative significant correlations were found between mean right AT and mean right SDS $(r=-0.91, \mathrm{p}<0.01$, Bonferroni corrected) and between mean right SDS and THI $(\mathrm{r}=-0.81, \mathrm{p}<0.01$, Bonferroni corrected) (Table 1$)$. No correlation was found between left ear mean AT and SDS of ISSNHL patients and HC subjects' audiological scores.

\subsection{PET/CT examination}

As depicted in Table 2 and Fig. 2 ISSNHL patients demonstrated a significant relative increase of glucose metabolism in the right superior (Brodmann's Areas; BA10) and medial (BA9) frontal gyrus as well as in the right anterior cingulate cortex (BA24, 32) compared to HC subjects. In the opposite comparison, ISSNHL showed a significant relative decrease of glucose metabolism in the bilateral precentral (BA4, 6), right middle temporal (BA19) and postcentral (BA1) gyrus as well as in the left posterior cingulate (BA31) cortex, left lingual (BA19), superior (BA22, 41) and middle (BA21) temporal and middle frontal gyrus (BA6) and in the left insula (BA13) (Table 2, Fig. 2).

In ISSNHL patients, SPM regression analysis showed a positive correlation between mean THI and glucose consumption in the right anterior cingulate cortex of BAs 24 and 32 (Table 3, Fig. 3). Furthermore, a positive correlation between mean SDS and glucose consumption in the left precentral gyrus of BA4 and 6 and left insula (BA13) was found (Table 3, Fig. 3).

\section{Discussion}

Considering the lack of findings in this field (Boyen et al., 2013; Husain et al., 2011; Suzuki et al., 2002; Yang et al., 2014), the present study aimed at assessing early stages of cortical metabolic changes following ISSNHL by using a resting state PET technique in order to further examine the phenomena of plasticity possibly affecting the 'baseline' metabolic condition of the brain. The major findings highlighted here reside in the relative hypometabolism depicted in the contralateral auditory (left BA21, 22, 41), in the bilateral associative visual (BA 19) and left posterior cingulate cortex (BA31), in the left insula (BA13), bilateral sensory-motor $(B A 1,4)$ and supplementary motor area (SMA; BA6) in ISSNHL patients compared to in $\mathrm{HC}$ subjects. Conversely, relative hypermetabolism in ISSNHL subjects compared to in HC subjects in prefrontal (PFC; BA9,10) and anterior cingulate cortex (ACC; BA24/ 32 ) was found during the acute stage of disease (Table 2, Fig. 2).

\subsection{Metabolic decrease in auditory and associative areas}

The relative reduction of the metabolism in the contralateral auditory cortex in the present study could be the metabolic

Table 1

Significant correlation scores between audiological tests.

\begin{tabular}{llll}
\hline & Mean AT & Mean SDS & THI \\
\hline Mean AT & 1 & & \\
Mean SDS & -0.91 & 1 & \\
THI & 0.95 & -0.81 & 1 \\
\hline
\end{tabular}

Significant $r$ values for positive correlation found between mean AT (auditory threshold) and THI (Tinnitius Handicap Inventory) and for negative correlations found between mean AT and mean SDS (speech discrimination score) and between mean SDS and THI. counterpart of ipsilateral hemispheric dominance found in previous functional studies (Suzuki et al., 2002) and contralateral grey matter reductions postulated in a recent resting-state neuroimaging study performed during the acute stage of ISSNHL (Fan et al., 2015). According to these authors, the hypothesized mechanism behind this kind of impairment could be related to the misrepresentation of the perception of intensity at the cortical level (Musiek et al., 2013), where it could be affected by the interaction between excitatory and inhibitory neurons (Fan et al., 2015; Musiek et al., 2013; Phillips et al., 1994). Interestingly, this decrease in grey matter volume (GMV) was found to correlate negatively with disease duration, which is in line with the studies in which a GMV reduction was indeed found in the chronic phase of the disease (Boyen et al., 2013; Yang et al., 2014).

These findings could further reinforce the relative cerebral glucose metabolism (rCGM) or blood flow decrease found in the auditory cortex and in many associative areas in previous studies on patients with chronic deafness and tinnitus (Okuda et al., 2013; Ueyama et al., 2015; Lanting et al., 2009), suggesting that reorganizational behaviour also occurs during ISSNHL. One hierarchical organization of primary and associative auditory cortex (Ruytjens et al., 2006) involves carrying out demanding neural computations, performed automatically and efficiently by the normal brain. This "auditory scene analysis" network has been shown to engage brain mechanisms in the auditory association cortex in the posterior superior temporal gyrus and its connections (Altmann et al., 2008; Brunetti et al., 2008; Golden et al., 2016; Warren and Griffiths, 2003; Zimmer et al., 2006), including the posterior cingulate, precuneus and retrosplenial cortex (Golden et al., 2016; Leech and Sharp, 2014).

\subsection{Metabolic arrangement in the default mode network, insula and frontoparietal control system}

Many of the above-mentioned areas have been identified in order to overlap neuroanatomical correlates, included in the core regions of the so-called "default-mode network" (DMN): a brain network linking mesial temporal, lateral parietal, and prefrontal regions via a posterior medial cortical hub zone (Fransson and Marrelec, 2008; Micarelli et al., 2013; Raichle et al., 2001; Shulman et al., 1997).

In this scenario the insula - which is not entangled in the DMN is likely to act as a multimodal hub that integrates body state information with incoming sensory traffic from the external environment (Golden et al., 2016; Husain and Schmidt, 2014). This region is therefore in a position where it can link the DMN with the brain networks that evaluate sensory stimuli and program behavioural responses (Seeley et al., 2009; Zhou and Seeley, 2014). Considering its multiple (however not fully defined) functions, insular rCGM decrease here might be thought to reflect a "freezing" behaviour in a multimodal region involved in processing demanding sensory traffic during auditory scene changes in which an unilateral auditory modality loss has abruptly occurred (Golden et al., 2016).

On the other hand, a rCGM increase was found in PFC as well as in ACC in ISSNHL patients but not in HC subjects. Previous metabolic studies (Schecklmann et al., 2013) suggested that this constellation of regions, which was found to be involved in the socalled "frontoparietal control system" (Medalla and Barbas, 2014), is commonly engaged by tasks that require controlled processing of information (Botvinick, 2007; Crone et al., 2006; Dosenbach et al., 2007), and this area is closely connected to limbic areas involved in the autonomic responses and in pathophysiology of tinnitus (Schecklmann et al., 2013). For instance, the ACC was found to commonly increase activity during conflict monitoring (Botvinick, 
Table 2

Numerical results of SPM comparisons between ${ }^{18}$ F-FDG uptake in ISSNHL $(\mathrm{N}=14)$ and HC $(\mathrm{n}=36)$.

\begin{tabular}{|c|c|c|c|c|c|c|c|}
\hline \multirow[t]{2}{*}{ Comparison } & \multicolumn{3}{|l|}{ Cluster level } & \multicolumn{4}{|l|}{ Voxel level } \\
\hline & Cluster extent & Cluster p (FDR-corr) & Cluster p (FWE corr) & Z score of maximum & Talairach coordinates & Cortical region & $\mathrm{BA}$ \\
\hline \multirow[t]{5}{*}{ ISSNHL-HC } & 5947 & 0.000 & 0.000 & 6.37 & $10,70,6$ & Right superior frontal gyrus & 10 \\
\hline & & & & 6.23 & $-2,56,40$ & Right medial frontal gyrus & 9 \\
\hline & 1286 & 0.000 & 0.000 & 5.83 & $12,28,16$ & Right anterior cingulate & 24 \\
\hline & & & & 5.59 & $20,14,34$ & Right cingulate gyrus & 32 \\
\hline & & & & 5.47 & $14,22,22$ & Right anterior cingulate & 32 \\
\hline \multirow[t]{13}{*}{ HC-ISSNHL } & 18022 & 0.000 & 0.000 & 7.37 & $62,-66,18$ & Right middle temporal gyrus & 19 \\
\hline & & & & 6.51 & $-10,-66,14$ & Left posterior cingulate & 31 \\
\hline & & & & 6.08 & $-12,-58,0$ & Left lingual gyrus & 19 \\
\hline & 2565 & 0.000 & 0.000 & 5.99 & $34,-22,72$ & Right precentral gyrus & 4 \\
\hline & & & & 5.76 & $56,-24,60$ & Right postcentral gyrus & 1 \\
\hline & & & & 5.62 & $46,-2,48$ & Right precentral gyrus & 6 \\
\hline & 731 & 0.001 & 0.001 & 5.50 & $-48,-26,6$ & Left superior temporal gyrus & 41 \\
\hline & & & & 5.45 & $-48,-34,20$ & Left insula & 13 \\
\hline & 249 & 0.041 & 0.035 & 5.09 & $-68,-28,4$ & Left superior temporal gyrus & 22 \\
\hline & & & & 4.45 & $-68,-42,0$ & Left middle temporal gyrus & 21 \\
\hline & 960 & 0.000 & 0.000 & 4.90 & $-38,-6,42$ & Left middle frontal gyrus & 6 \\
\hline & & & & 4.14 & $-34,2,56$ & Left middle frontal gyrus & 6 \\
\hline & & & & 4.09 & $-32,-18,42$ & Left precentral gyrus & 4 \\
\hline
\end{tabular}

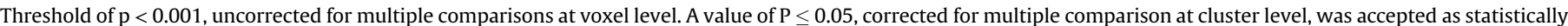

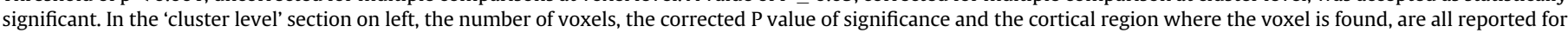

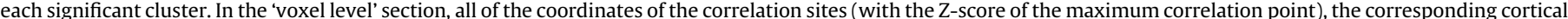

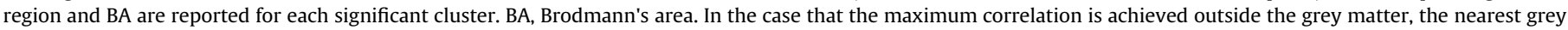
matter (within a range of $7 \mathrm{~mm}$ ) is indicated with the corresponding BA.

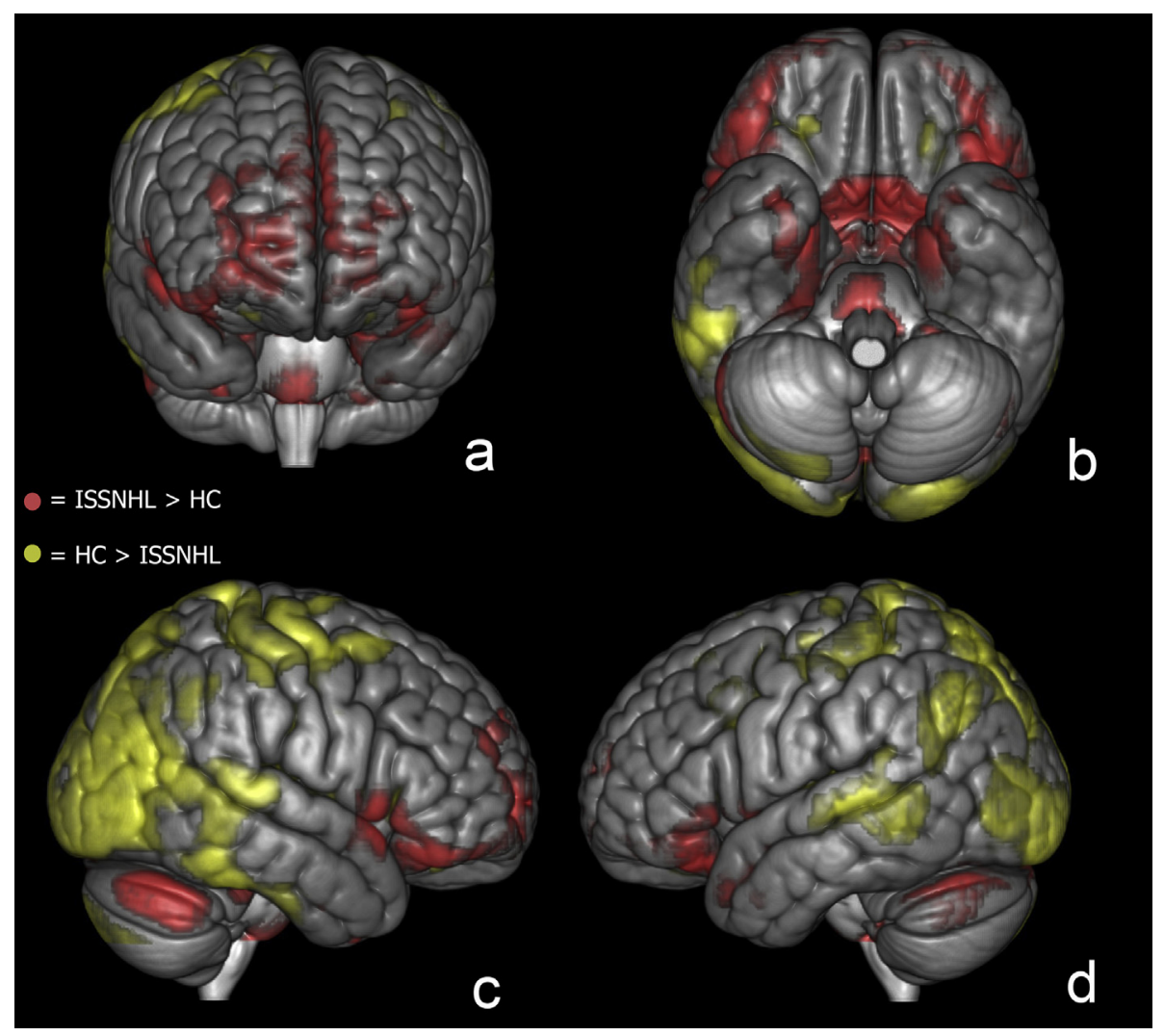

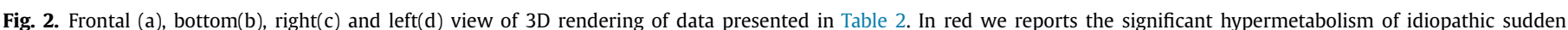

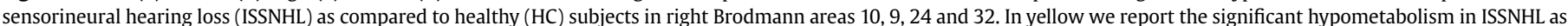

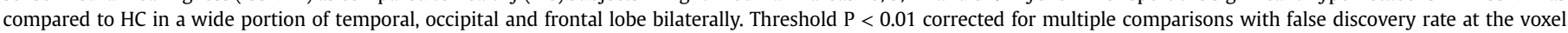

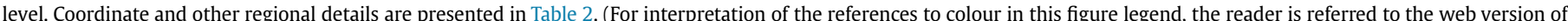
this article.)

2007; Dosenbach et al., 2006, 2007) and the PFC has been associated with the coordination of information processing and information transfer between multiple brain regions during the performance of concurrent tasks (Ramnani and Owen, 2004; Medalla and Barbas, 2014). In particular, the frontopolar area BA10 - previously dubbed frontal "auditory field" (Medalla and 
Table 3

Numerical results of SPM regression analysis between ${ }^{18}$ F-FDG uptake in ISSNHL $(\mathrm{N}=14)$, THI and SDS.

\begin{tabular}{|c|c|c|c|c|c|c|c|}
\hline \multirow[t]{2}{*}{ Comparison } & \multicolumn{3}{|l|}{ Cluster level } & \multicolumn{4}{|l|}{ Voxel level } \\
\hline & Cluster extent & Cluster p (FDR-corr) & Cluster p (FWE corr) & $\mathrm{Z}$ score of maximum & Talairach coordinates & Cortical region & BA \\
\hline \multirow[t]{3}{*}{+ THI } & 334 & 0.014 & 0.016 & 5.92 & $-6,28,16$ & Left anterior cingulate & 24 \\
\hline & & & & 5.39 & $12,34,22$ & Right anterior cingulate & 32 \\
\hline & & & & 5.11 & $6,28,10$ & Right anterior cingulate & 24 \\
\hline \multirow[t]{5}{*}{+ SDS } & & & & 5.88 & $-46,-10,42$ & Left precentral gyrus & 4 \\
\hline & & & & 5.79 & $-62,-10,32$ & Left precentral gyrus & 6 \\
\hline & & & & 5.18 & $-38,-20,66$ & Left precentral gyrus & 6 \\
\hline & & & & 4.91 & $-32,-12,70$ & Left precentral gyrus & 6 \\
\hline & & & & 5.04 & $33,-52,-42$ & Left superior temporal gyrus & 13 \\
\hline
\end{tabular}

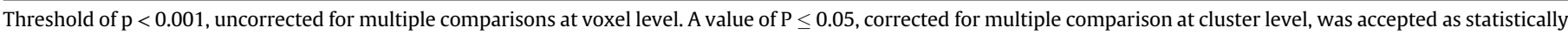

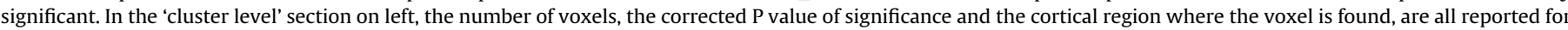

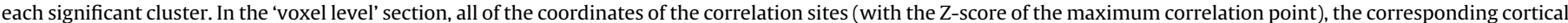

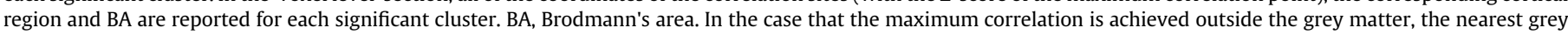
matter (within a range of $7 \mathrm{~mm}$ ) is indicated with the corresponding BA. Tinnitus Handicap Inventory, THI; Speech Discrimination Score, SDS.

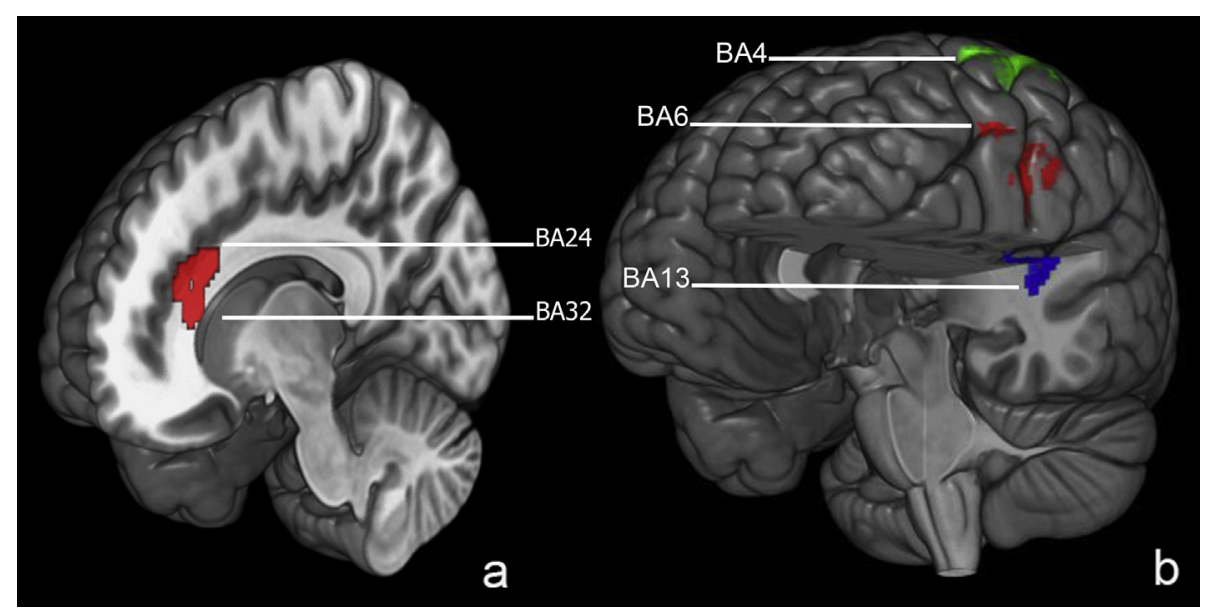

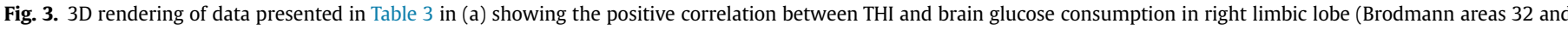

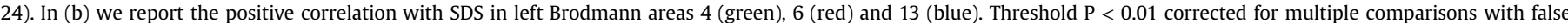

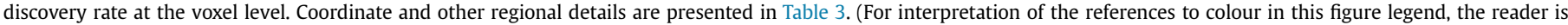
referred to the web version of this article.)

Barbas, 2014) - demonstrated rich and varied bidirectional connections with auditory association cortices (Medalla and Barbas, 2014). In this light and in line with previous metabolic findings (Berding et al., 2015), the rCGM increase found during the acute stage of ISSNHL could be interpreted as an attempt in conflict monitoring and in informative trace integration coming from sensory error detection, such as those represented by an unexpected unilateral auditory input impairment. Furthermore, although no lateralization of rCGM increase in auditory cortex was found in this study, compared to previous studies (Arnold et al., 1996; Wang et al., 2001; Langguth et al., 2006), the present findings could further reinforce the hypotheses that postulate activity changes in PFC and ACC as encompassed in the tinnitus network (Schecklmann et al., 2013; Lanting et al., 2009).

\subsubsection{Correlation analysis}

These aspects are further corroborated by the positive correlation found between right ACC and THI scores (Table 3, Fig. 3). In fact, considering the debated mechanistic origin of tinnitus, it is unclear whether hyperactivity along the auditory pathway is a direct consequence of cochlear cell damage or whether it results from hyperactivity in other neuronal pathways (Jastreboff, 1990; Job et al., 2012; Roberts et al., 2010; Xu et al., 2016). The abovementioned positive correlation between THI scores and ACC could further highlight the theories that concentrate on bidirectional influences between tinnitus and sub-components of a general emotional limbic network (e.g. ACC was previously identified as part of the tinnitus affective network) (Adamchic et al., 2014; Job et al., 2012; Roberts et al., 2010; Vanneste et al., 2010). This could indicate that the activation of ACC - due to a general feature of stress responses and its wide connections with auditory system and associative areas (Job et al., 2012; Medalla and Barbas, 2014; Xu et al., 2016) - may be concurrent in tinnitus triggering also during acute stages of sudden loss of auditory inflow.

A further interesting finding is the positive correlation between mean SDS scores and rCGM found in left BA4/6 and left insula (Table 3, Fig. 3). These areas were found to be affected by a relatively decreased metabolism in ISSNHL patients, compared to healthy subjects and SDS scores were also found to be significantly reduced in the affected ear. In addition to the anatomical connections with Besides the fact that confined anatomical grounds are depicted for the wide range of "higher" functions of these regions (Nachev et al., 2007), these structures were identified as commonly involved in the case of degraded speech processing, considering that discrimination is clearly not specific to auditory envelope processing, but has been suggested to be involved rather in top-down executive processes (Erb et al., 2013).

Since previous studies demonstrated these regions (Eckert et al., 2009) as engaged when tasks become increasingly difficult, their involvement was found to be pivotal for a wide range of challenging 
listening situations, such as when listening to speech (Erb et al., 2013; Wild et al., 2012).

Considering that experimental data were gathered in absence of controlled sound stimulation, the relative rCGM decrease found in these regions and the positive correlation with mean SDS scores in ISSNHL could highlight new aspects of the plasticity of these areas. This could contribute to further explain changes in the executive functions underpinning speech recognition during the sudden impairment of unilateral auditory input.

Finally, a significant positive correlation was found between mean AT and THI as well as negative correlations between mean AT and mean SDS and between mean SDS and THI (Table 1). This confirms the association between loss of auditory input, tinnitus onset and speech discrimination impairment in the acute phase of the disease (Muhlmeier et al., 2016).

\section{Conclusions}

The present study might be the first attempt to further examine the neuroanatomical metabolic changes that may induce the clinical-perceptual rearrangements occurring at the very early stage of unilateral auditory impairment. However, a possible limitation of the study could be related to the small sample size, increasing the likelihood of Type II statistical errors. In fact, acute otoneurological patients, due to their very severe symptomatology, often (Alessandrini et al., 2013, 2014b) refused or delayed the PET examination due to the discomfort in lying still in the gantry, which, together with the relative high costs of the PET/CT methodology, makes the recruitment of an adequate number of subjects to be investigated a common limitation in functional neuroimaging studies.

Finally, a possible limitation of our study could be the choice of the cerebellum for the normalization of PET data used later for regression analyses with audiological tests (Table 3 ). In a study using manganese enhanced MRI, Brozoski et al. reported the changes in the cerebellum of animals with tinnitus. In particular, the authors found that in rats with evidence of tinnitus, activity was generally elevated in the auditory brainstem, with significant elevation in the cerebellar paraflocculus, the posterior ventral cochlear nucleus, and the inferior colliculus (Brozoski et al., 2007). Chen et al. reported changes in the cerebellum of rats shortly after tinnitus was induced with a high dose of salicylate (Chen et al., 2014). This evidence could limit the use of the cerebellum for normalization, resulting in a sub-optimal structure for data normalization in certain circumstances and in particular when variations in brain activity in this structure are detectable during tasks or pathological conditions. On the other hand, as pointed out in Table 2, we did not find significant differences when comparing ISSNHL vs. HC and vice versa in the cerebellum thus supporting the choice of this subcortical structure for the normalization of PET data for regression analyses. These aspects could be kept in mind when devising future research for acquiring more insights into early and delayed brain metabolic changes of larger sample sizes of ISSNHL patients., also considering the clinical outcomes of the patients enrolled in the present study (Table 4, Supplementary Material).

\section{Authors' contribution}

Marco Alessandrini, Alessandro Micarelli, Orazio Schillaci and Andrea Viziano conceived and designed the experiment; Marco Alessandrini, Alessandro Micarelli, Andrea Viziano and Agostino Chiaravalloti performed the experiment; Agostino Chiaravalloti, Alessandro Micarelli, Marco Alessandrini and Andrea Viziano analysed the data; Agostino Chiaravalloti, Marco Alessandrini, Roberta
Danieli and Orazio Schillaci contributed clinical data/materials/ analysis tools; Alessandro Micarelli, Andrea Viziano, Marco Alessandrini and Agostino Chiaravalloti wrote the paper.

\section{Conflict of interest/financial support}

There is no conflict of interest in this study.

\section{Appendix A. Supplementary data}

Supplementary data related to this article can be found at http:// dx.doi.org/10.1016/j.heares.2017.04.011.

\section{References}

Adamchic, I., Langguth, B., Hauptmann, C., Tass, P.A., 2014. Abnormal crossfrequency coupling in the tinnitus network. Front. Neurosci. 8, 284.

Alessandrini, M., Micarelli, A., Chiaravalloti, A., Candidi, M., Bruno, E., Di Pietro, B., Schillaci, O., Pagani, M., 2014a. Cortico-subcortical metabolic correlates of olfactory processing in healthy resting subjects. Sci. Rep. 4, 5146.

Alessandrini, M., Pagani, M., Napolitano, B., Micarelli, A., Candidi, M., Bruno, E., Chiaravalloti, A., Di Pietro, B., Schillaci, O., 2013. Early and phasic cortical metabolic changes in vestibular neuritis onset. PloS One 8, e57596.

Alessandrini, M., Micarelli, A., Chiaravalloti, A., Candidi, M., Bruno, E., Di Pietro, B., Oberg, J., Schillaci, O., Pagani, M., 2014b. Cerebellar metabolic involvement and its correlations with clinical parameters in vestibular neuritis. J. Neurol. 261, 1976-1985.

Alessandrini, M., Micarelli, A., Chiaravalloti, A., Bruno, E., Danieli, R., Pierantozzi, M., Genovesi, G., Oberg, J., Pagani, M., Schillaci, O., 2016. Involvement of subcortical brain structures during olfactory stimulation in multiple chemical sensitivity. Brain Topogr. 29, 243-252.

Alexander, T.H., Harris, J.P., 2013. Incidence of sudden sensorineural hearing loss. Otol. Neurotol. Off. Pub. Am. Otol. Soc. Am. Neurotol. Soc. Eur. Acad. Otol. Neurotol. 34, 1586-1589.

Altmann, C.F., Henning, M., Doring, M.K., Kaiser, J., 2008. Effects of feature-selective attention on auditory pattern and location processing. Neurolmage 41, 69-79.

Arnold, W., Bartenstein, P., Oestreicher, E., Romer, W., Schwaiger, M., 1996. Focal metabolic activation in the predominant left auditory cortex in patients suffering from tinnitus: a PET study with $\left[{ }^{18}\right.$ F $]$ deoxyglucose. ORL J. Oto-rhinolaryngol. Relat. Spec. 58, 195-199.

Benjamin, C., Lieberman, D.A., Chang, M., Ofen, N., Whitfield-Gabrieli, S., Gabrieli, J.D., Gaab, N., 2010. The influence of rest period instructions on the default mode network. Front. Hum. Neurosci. 4, 218.

Bennett, C.M., Wolford, G.L., Miller, M.B., 2009. The principled control of false positives in neuroimaging. Soc. Cogn. Affect. Neurosci. 4, 417-422.

Berding, G., Wilke, F., Rode, T., Haense, C., Joseph, G., Meyer, G.J., Mamach, M., Lenarz, M., Geworski, L., Bengel, F.M., Lenarz, T., Lim, H.H., 2015. Positron emission tomography imaging reveals auditory and frontal cortical regions involved with speech perception and loudness adaptation. PloS One 10, e0128743.

Berrettini, S., Seccia, V., Fortunato, S., Forli, F., Bruschini, L., Piaggi, P., Canapicchi, R., 2013. Analysis of the 3-dimensional fluid-attenuated inversion-recovery (3DFLAIR) sequence in idiopathic sudden sensorineural hearing loss. JAMA Otolaryngol. Head Neck Surg. 139, 456-464.

Bilecen, D., Seifritz, E., Radu, E.W., Schmid, N., Wetzel, S., Probst, R., Scheffler, K., 2000. Cortical reorganization after acute unilateral hearing loss traced by fMRI. Neurology 54, 765-767.

Botvinick, M.M., 2007. Conflict monitoring and decision making: reconciling two perspectives on anterior cingulate function. Cogn. Affect. Behav. Neurosci. 7, $356-366$.

Boyen, K., Langers, D.R., de Kleine, E., van Dijk, P., 2013. Gray matter in the brain: differences associated with tinnitus and hearing loss. Hear. Res. 295, 67-78.

Brozoski, T.J., Ciobanu, L., Bauer, C.A., 2007. Central neural activity in rats with tinnitus evaluated with manganese-enhanced magnetic resonance imaging (MEMRI). Hear. Res. 228, 168-179.

Brunetti, M., Della Penna, S., Ferretti, A., Del Gratta, C., Cianflone, F., Belardinelli, P., Caulo, M., Pizzella, V., Olivetti Belardinelli, M., Romani, G.L., 2008. A frontoparietal network for spatial attention reorienting in the auditory domain: a human fMRI/MEG study of functional and temporal dynamics. Cereb. Cortex 18, 1139-1147 (New York, N.Y: 1991).

Capuano, L., Cavaliere, M., Parente, G., Damiano, A., Pezzuti, G., Lopardo, D. Iemma, M., 2015. Hyperbaric oxygen for idiopathic sudden hearing loss: is the routine application helpful? Acta Oto-laryngol. 135, 692-697.

Cardier, M., Zulueta-Santos, C., Manrique-Huarte, R., Prieto, E., Garcia-Garcia, B., Arbizu, J., Manrique, M., 2015. Functional neuroimaging studies in asymmetric hearing loss. Audiol. Neuro-otol. 20 (Suppl. 1), 48-52.

Chang, Y., Lee, S.H., Lee, Y.J., Hwang, M.J., Bae, S.J., Kim, M.N., Lee, J., Woo, S., Lee, H., Kang, D.S., 2004. Auditory neural pathway evaluation on sensorineural hearing loss using diffusion tensor imaging. Neuroreport 15, 1699-1703.

Chen, G., Feng, L., Liu, Z., Sun, Y., Chang, H., Cui, P., 2014. Both central and peripheral 
auditory systems are involved in salicylate-induced tinnitus in rats: a behavioral study. PloS One 9, e108659.

Chiaravalloti, A., Pagani, M., Micarelli, A., Di Pietro, B., Genovesi, G., Alessandrini, M., Schillaci, O., 2015. Cortical activity during olfactory stimulation in multiple chemical sensitivity: a (18)F-FDG PET/CT study. Eur. J. Nucl. Med. Mol. Imaging 42, 733-740.

Chiaravalloti, A., Pagani, M., Di Pietro, B., Danieli, R., Tavolozza, M., Travascio, L., Caracciolo, C.R., Simonetti, G., Cantonetti, M., Schillaci, O., 2013. Is cerebral glucose metabolism affected by chemotherapy in patients with Hodgkin's lymphoma? Nucl. Med. Commun. 34, 57-63.

Crone, E.A., Wendelken, C., Donohue, S.E., Bunge, S.A., 2006. Neural evidence for dissociable components of task-switching. Cereb. Cortex 16, 475-486 (New York, N.Y. : 1991).

Della Rosa, P.A., Cerami, C., Gallivanone, F., Prestia, A., Caroli, A., Castiglioni, I., Gilardi, M.C., Frisoni, G., Friston, K., Ashburner, J., Perani, D., 2014. A standardized $\left[{ }^{18} \mathrm{~F}\right]$-FDG-PET template for spatial normalization in statistical parametric mapping of dementia. Neuroinformatics 12, 575-593.

Dosenbach, N.U., Visscher, K.M., Palmer, E.D., Miezin, F.M., Wenger, K.K., Kang, H.C., Burgund, E.D., Grimes, A.L., Schlaggar, B.L., Petersen, S.E., 2006. A core system for the implementation of task sets. Neuron 50, 799-812.

Dosenbach, N.U., Fair, D.A., Miezin, F.M., Cohen, A.L., Wenger, K.K., Dosenbach, R.A., Fox, M.D., Snyder, A.Z., Vincent, J.L., Raichle, M.E., Schlaggar, B.L., Petersen, S.E., 2007. Distinct brain networks for adaptive and stable task control in humans. Proc. Natl. Acad. Sci. U. S. A. 104, 11073-11078.

Eckert, M.A., Menon, V., Walczak, A., Ahlstrom, J., Denslow, S., Horwitz, A., Dubno, J.R., 2009. At the heart of the ventral attention system: the right anterior insula. Hum. Brain Mapp. 30, 2530-2541.

Erb, J., Henry, M.J., Eisner, F., Obleser, J., 2013. The brain dynamics of rapid perceptual adaptation to adverse listening conditions. J. Neurosci. Off. J. Soc. Neurosci. 33, 10688-10697.

Fan, W., Zhang, W., Li, J., Zhao, X., Mella, G., Lei, P., Liu, Y., Wang, H., Cheng, H., Shi, H., $\mathrm{Xu}, \mathrm{H} ., 2015$. Altered contralateral auditory cortical morphology in unilateral sudden sensorineural hearing loss. Otol. Neurotol. Off. Pub. Am. Otol. Soc. Am. Neurotol. Soc. Eur. Acad. Otol. Neurotol. 36, 1622-1627.

Frackowiak, R.S., Friston, K.J., 1994. Functional neuroanatomy of the human brain: positron emission tomography-a new neuroanatomical technique. J. Anat. 184 (Pt 2), 211-225.

Fransson, P., Marrelec, G., 2008. The precuneus/posterior cingulate cortex plays a pivotal role in the default mode network: evidence from a partial correlation network analysis. NeuroImage 42, 1178-1184.

Gaab, N., Gabrieli, J.D., Glover, G.H., 2008. Resting in peace or noise: scanner background noise suppresses default-mode network. Hum. Brain Mapp. 29, $858-867$.

Golden, H.L., Agustus, J.L., Nicholas, J.M., Schott, J.M., Crutch, S.J., Mancini, L., Warren, J.D., 2016. Functional neuroanatomy of spatial sound processing in Alzheimer's disease. Neurobiol. Aging 39, 154-164.

Husain, F.T., Medina, R.E., Davis, C.W., Szymko-Bennett, Y., Simonyan, K., Pajor, N.M., Horwitz, B., 2011. Neuroanatomical changes due to hearing loss and chronic tinnitus: a combined VBM and DTI study. Brain Res. 1369, 74-88.

Husain, F.T., Schmidt, S.A., 2014. Using resting state functional connectivity to unravel networks of tinnitus. Hear. Res. 307, 153-162.

Jastreboff, P.J., 1990. Phantom auditory perception (tinnitus): mechanisms of generation and perception. Neurosci. Res. 8, 221-254.

Job, A., Pons, Y., Lamalle, L., Jaillard, A., Buck, K., Segebarth, C., Delon-Martin, C., 2012. Abnormal cortical sensorimotor activity during "Target" sound detection in subjects with acute acoustic trauma sequelae: an fMRI study. Brain Behav. 2, 187-199.

Lancaster, J.L., Rainey, L.H., Summerlin, J.L., Freitas, C.S., Fox, P.T., Evans, A.C., Toga, A.W., Mazziotta, J.C., 1997. Automated labeling of the human brain: a preliminary report on the development and evaluation of a forward-transform method. Hum. Brain Mapp. 5, 238-242.

Lancaster, J.L., Woldorff, M.G., Parsons, L.M., Liotti, M., Freitas, C.S., Rainey, L., Kochunov, P.V., Nickerson, D., Mikiten, S.A., Fox, P.T., 2000. Automated Talairach atlas labels for functional brain mapping. Hum. Brain Mapp. 10, 120-131.

Langguth, B., Eichhammer, P., Kreutzer, A., Maenner, P., Marienhagen, J., Kleinjung, T., Sand, P., Hajak, G., 2006. The impact of auditory cortex activity on characterizing and treating patients with chronic tinnitus-first results from a PET study. Acta Oto-laryngol. 84-88. Supplementum.

Lanting, C.P., de Kleine, E., van Dijk, P., 2009. Neural activity underlying tinnitus generation: results from PET and fMRI. Hear. Res. 255, 1-13.

Leech, R., Sharp, D.J., 2014. The role of the posterior cingulate cortex in cognition and disease. Brain J. Neurol. 137, 12-32.

Liguori, C., Chiaravalloti, A., Sancesario, G., Stefani, A., Sancesario, G.M., Mercuri, N.B., Schillaci, O., Pierantozzi, M., 2016. Cerebrospinal fluid lactate levels and brain $\left[{ }^{18} \mathrm{~F}\right] \mathrm{FDG}$ PET hypometabolism within the default mode network in Alzheimer's disease. Eur. J. Nucl. Med. Mol. Imaging 43, 2040-2049.

Lin, Y., Wang, J., Wu, C., Wai, Y., Yu, J., Ng, S., 2008. Diffusion tensor imaging of the auditory pathway in sensorineural hearing loss: changes in radial diffusivity and diffusion anisotropy. J. Magn. Reson. Imaging JMRI 28, 598-603.

Maldjian, J.A., Laurienti, P.J., Kraft, R.A., Burdette, J.H., 2003. An automated method for neuroanatomic and cytoarchitectonic atlas-based interrogation of fMRI data sets. Neurolmage 19, 1233-1239.

Medalla, M., Barbas, H., 2014. Specialized prefrontal "auditory fields": organization of primate prefrontal-temporal pathways. Front. Neurosci. 8, 77.

Micarelli, A., Jacobsson, H., Larsson, S.A., Jonsson, C., Pagani, M., 2013.
Neurobiological insight into hyperbaric hyperoxia. Acta physiol. Oxf. Engl. 209, 69-76.

Micarelli, A., Viziano, A., Genovesi, G., Bruno, E., Ottaviani, F., Alessandrini, M., 2016. Lack of contralateral suppression in transient-evoked otoacoustic emissions in multiple chemical sensitivity: a clinical correlation study. Noise Health 18 , $143-149$.

Monzani, D., Genovese, E., Marrara, A., Gherpelli, C., Pingani, L., Forghieri, M., Rigatelli, M., Guadagnin, T., Arslan, E., 2008. Validity of the Italian adaptation of the Tinnitus Handicap Inventory; focus on quality of life and psychological distress in tinnitus-sufferers. Acta Otorhinolaryngol. Ital. Organo Uff. Soc. Ital. Otorinolaringol. Chir. Cerv.-Facc. 28, 126-134.

Muhlmeier, G., Baguley, D., Cox, T., Suckfull, M., Meyer, T., 2016. Characteristics and spontaneous recovery of tinnitus related to idiopathic sudden sensorineural hearing loss. Otol. Neurotol. Off. Publ. Am. Otol. Soc. Am. Neurotol. Soc. Eur. Acad. Otol. Neurotol. 37, 634-641.

Musiek, F., Guenette, L., Fitzgerald, K., 2013. Lateralized auditory symptoms in central neuroaudiology disorder. J. Am. Acad. Audiol. 24, 556-563.

Nachev, P., Wydell, H., O'Neill, K., Husain, M., Kennard, C., 2007. The role of the presupplementary motor area in the control of action. NeuroImage 36 (Suppl. 2), T155-T163.

Okuda, T., Nagamachi, S., Ushisako, Y., Tono, T., 2013. Glucose metabolism in the primary auditory cortex of postlingually deaf patients: an FDG-PET study. ORL J. oto-rhino-laryngol. Relat. Spec. 75, 342-349.

Pagani, M., De Carli, F., Morbelli, S., Oberg, J., Chincarini, A., Frisoni, G.B., Galluzzi, S., Perneczky, R., Drzezga, A., van Berckel, B.N., Ossenkoppele, R., Didic, M., Guedj, E., Brugnolo, A., Picco, A., Arnaldi, D., Ferrara, M., Buschiazzo, A., Sambuceti, G., Nobili, F., 2015. Volume of interest-based $\left[{ }^{18}\right.$ F fluorodeoxyglucose PET discriminates MCI converting to Alzheimer's disease from healthy controls. A European Alzheimer's Disease Consortium (EADC) study. NeuroImage. Clin. 7, 34-42.

Penrod, J.P., 1994. Speech threshold and word recognition/discrimination testing In: Katz, J. (Ed.), Handbook of Clinical Audiology, fourth ed. Williams and Wilkins, Baltimore.

Phillips, D.P., Semple, M.N., Calford, M.B., Kitzes, L.M., 1994. Level-dependent representation of stimulus frequency in cat primary auditory cortex. Exp. Brain Res. 102, 210-226.

Profant, O., Skoch, A., Balogova, Z., Tintera, J., Hlinka, J., Syka, J., 2014. Diffusion tensor imaging and MR morphometry of the central auditory pathway and auditory cortex in aging. Neuroscience 260, 87-97.

Raichle, M.E., MacLeod, A.M., Snyder, A.Z., Powers, W.J., Gusnard, D.A., Shulman, G.L., 2001. A default mode of brain function. Proc. Natl. Acad. Sci. U. S. A. $98,676-682$.

Ramnani, N., Owen, A.M., 2004. Anterior prefrontal cortex: insights into function from anatomy and neuroimaging. Nature reviews. Neuroscience 5, 184-194.

Roberts, L.E., Eggermont, J.J., Caspary, D.M., Shore, S.E., Melcher, J.R., Kaltenbach, J.A., 2010. Ringing ears: the neuroscience of tinnitus. J. Neurosci. Off. J. Soc. Neurosci. 30, 14972-14979.

Ruytjens, L., Willemsen, A.T., Van Dijk, P., Wit, H.P., Albers, F.W., 2006. Functional imaging of the central auditory system using PET. Acta Oto-laryngol. 126, 1236-1244.

Seeley, W.W., Crawford, R.K., Zhou, J., Miller, B.L., Greicius, M.D., 2009. Neurodegenerative diseases target large-scale human brain networks. Neuron 62, $42-52$.

Schecklmann, M., Landgrebe, M., Poeppl, T.B., Kreuzer, P., Manner, P., Marienhagen, J., Wack, D.S., Kleinjung, T., Hajak, G., Langguth, B., 2013. Neural correlates of tinnitus duration and distress: a positron emission tomography study. Hum. Brain Mapp. 34, 233-240.

Shulman, G.L., Fiez, J.A., Corbetta, M., Buckner, R.L., Miezin, F.M., Raichle, M.E., Petersen, S.E., 1997. Common blood flow changes across visual tasks: II. Decreases in cerebral cortex. J. Cogn. Neurosci. 9, 648-663.

Soonawala, D., Amin, T., Ebmeier, K.P., Steele, J.D., Dougall, N.J., Best, J., Migneco, O., Nobili, F., Scheidhauer, K., 2002. Statistical parametric mapping of (99m)TcHMPAO-SPECT images for the diagnosis of Alzheimer's disease: normalizing to cerebellar tracer uptake. NeuroImage 17, 1193-1202.

Suzuki, M., Kouzaki, H., Nishida, Y., Shiino, A., Ito, R., Kitano, H., 2002. Cortical representation of hearing restoration in patients with sudden deafness. Neuroreport $13,1829-1832$.

Talavage, T.M., Gonzalez-Castillo, J., Scott, S.K., 2014. Auditory neuroimaging with fMRI and PET. Hear. Res. 307, 4-15.

Ueyama, T., Donishi, T., Ukai, S., Yamamoto, Y., Ishida, T., Tamagawa, S., Hotomi, M., Shinosaki, K., Yamanaka, N., Kaneoke, Y., 2015. Alterations of regional cerebral blood flow in tinnitus patients as assessed using single-photon emission computed tomography. PloS One 10, e0137291.

Vanneste, S., Van De Heyning, P., De Ridder, D., 2015. Tinnitus: a large VBM-EEG correlational study. PloS One 10, e0115122.

Vanneste, S., Plazier, M., van der Loo, E., Van de Heyning, P., De Ridder, D., 2010. The differences in brain activity between narrow band noise and pure tone tinnitus. Plos One 5, e13618.

Varrone, A., Asenbaum, S., Vander Borght, T., Booij, J., Nobili, F., Nagren, K., Darcourt, J., Kapucu, O.L., Tatsch, K., Bartenstein, P., Van Laere, K., 2009. EANM procedure guidelines for PET brain imaging using $\left[{ }^{18} \mathrm{~F}\right] \mathrm{FDG}$, version 2. Eur. J. Nucl. Med. Mol. Imaging 36, 2103-2110.

Wang, H., Tian, J., Yin, D., Jiang, S., Yang, W., Han, D., Yao, S., Shao, M., 2001. Regional glucose metabolic increases in left auditory cortex in tinnitus patients: a preliminary study with positron emission tomography. Chin. Med. J. 114, 848-851. 
Warren, J.D., Griffiths, T.D., 2003. Distinct mechanisms for processing spatial sequences and pitch sequences in the human auditory brain. J. Neurosci. Off. J. Soc. Neurosci. 23, 5799-5804.

Wild, C.J., Yusuf, A., Wilson, D.E., Peelle, J.E., Davis, M.H., Johnsrude, I.S., 2012. Effortful listening: the processing of degraded speech depends critically on attention. J. Neurosci. Off. J. Soc. Neurosci. 32, 14010-14021.

Xu, H., Fan, W., Zhao, X., Li, J., Zhang, W., Lei, P., Liu, Y., Wang, H., Cheng, H., Shi, H., 2016. Disrupted functional brain connectome in unilateral sudden sensorineural hearing loss. Hear. Res. 335, 138-148.
Yang, M., Chen, H.J., Liu, B., Huang, Z.C., Feng, Y., Li, J., Chen, J.Y., Zhang, L.L., Ji, H., Feng, X., Zhu, X., Teng, G.J., 2014. Brain structural and functional alterations in patients with unilateral hearing loss. Hear. Res. 316, 37-43.

Zhou, J., Seeley, W.W., 2014. Network dysfunction in Alzheimer's disease and frontotemporal dementia: implications for psychiatry. Biol. Psychiatry 75, 565-573.

Zimmer, U., Lewald, J., Erb, M., Karnath, H.O., 2006. Processing of auditory spatial cues in human cortex: an fMRI study. Neuropsychologia 44, 454-461. 